The 11 year-old woke with left-leg ataxia and sore neck, the morning after being tackled at a football game. He had continued playing to finish the game. Brain stem and cerebellar signs, including left exotropia with nystagmus, impaired adduction of the left eye in convergence, and deviation to the left in compass gait test, were explained by a linear filling defect of the left vertebral artery at C1-2 level consistent with intimal dissection, documented by a 4vessel cerebral arteriogram. CT and brain and cervical spine MRIs were normal. He recovered rapidly and was discharged to take one aspirin daily and avoid contact sports. The 8-year-old also recovered after minor neck trauma followed by recurrent episodes of ataxia, hemiparesis, and visual field defect with left VA thrombus at C-2 and multiple emboli in posterior cerebral arteries. The 7-year-old sustained a traumatic pseudoaneurysm with persistent hemiparesis and ataxia. Clinical data are summarized of 16 cases culled from the literature. (Garg BP, Fishman MA et al. Strokes in children due to

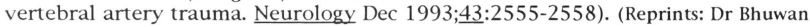
P Garg, Section of Child Neurology, Department of Neurology, Riley Children's Hospital, Rm 1757, 702 Barnhill Drive, Indianapolis, IN 46202).

COMMENT. Fortunately, the prognosis for survival in vertebral artery stroke in children is generally excellent. Only one of 19 patients died.

Two had residual quadriplegia, 9 had mild to moderate residual hemiparesis, ataxia, and/or dysarthria, and 7 (37\%) recovered.

\title{
OUTCOME OF NEONATAL STROKE
}

Evaluations, including MRI, MR angiography, and neuropsychological tests, at 1.5-8.4 years, of 8 infants after neonatal stroke involving the middle cerebral artery are reported from the University of Heidelberg, Mannheim, Germany. Seven had mental and motor retardation and hemiparesis and 4 had epilepsy. Major deficits in cognitive function were found in 4 older children. One patient with normal development showed only a localized temporal lobe lesion on MRI and unremarkable MRA. Children with marked disorders of motor and cognitive development had defects of temporo-parietal lobes, basal ganglia, thalamus and internal capsule on MRI and recanalization of the middle cerebral artery shown on MRA. Those without recanalization had a poorer prognosis. (Koelfen $\mathrm{W}$ et al. Results of parenchymal and angiographic magnetic resonance imaging and neuropsychological testing of children after stroke as neonates. Eur I Pediatr Dec 1993;152:1030-1035). (Respond: Dr W Koelfen,

Department of Pediatrics, Faculty of Clinical Medicine Mannheim, University of Heidelberg,Theodor Kutzer Ufer, D-68167 Mannheim, Germany).

COMMENT. Magnetic resonance angiography (MRA) images intracranial blood vessels without necessity for invasive contrast media. Children with neonatal stroke, seizures, and abnormalities shown on MRA have severe cognitive delays and the least favorable prognosis on long-term follow up.

\section{ASPIRIN-INDUCED NEONATAL INTRACRANIAL HEMORRHAGE}

A term newborn infant with intracranial hemorrhage associated with maternal acetylsalicylic acid ingestion before delivery is reported from the Departments of Pediatrics and Neurology, Eastern Virginia Medical School, Norfolk, VA. Pregnancy was complicated by alcohol abuse. Alka-Seltzer, 6 tablets daily, had been taken for 2 weeks for relief of hangover. The infant's serum salicylate level at 8 hours was $5.4 \mathrm{mg} / \mathrm{dL}$. Hematocrit decreased from 
$29 \%$ at birth to $15 \%$ at 6 hours of age. Prothrombin time was $>100$ sec. Cranial ultrasound showed hemorrhage in the tentorium and hydrocephalus. CT also revealed a large cerebellar hemorrhage. Clinical findings included multiple ecchymoses, gastric hemorrhage, hematuria, a tense fontanelle, head circumference at the 95th percentile, hypotonia, and ocular bobbing and nystagmus. Transfusion, a second injection of vitamin $K$, and ventriculoperitoneal shunt were followed by recovery and discharge in care of grandmother on day 23. (Karlowicz MG, White LE. Severe intracranial hemorrhage in a term neonate associated with maternal acetylsalicylic acid

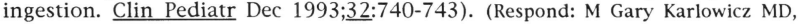
Department of Pediatrics, Eastern Virginia Medical School, Children's Hospital of The King's Daughters, 601 Children's Lane, Norfolk, VA 23507).

COMMENT. Maternal salicylate ingestion should be considered in the etiology of neonatal intracranial hemorrhage without birth trauma. The infant's relatively low salicylate level is not inconsistent with chronic toxicity.

Surfactant therapy for respiratory distress syndrome in newborns of $600-750 \mathrm{~g}$ birth weight caused an increased risk of grades I and II intracranial hemorrhage compared to controls, in analyses of the literature by researchers at Ross Laboratories, Columbus, OH (Gunkel JH, Banks PLC. Pediatrics Dec 1993;92:775-786).

\section{INFECTIOUS DISORDERS}

\section{LYME DISEASE NEUROLOGIC SYNDROMES}

The neurologic manifestations and syndromes associated with Lyme disease are reported in 96 patients, ages 3 to 19 years, living on Long Island and referred to University Hospital, Stony Brook, New York. All were seropositive for anti- $B$ burgdorferi antibodies. One-third had no prior history of extraneural manifestations of Lyme disease (erythema migrans, arthritis, flulike symptoms, and arthralgias/myalgias), and $90 \%$ had no memory of a tick bite. The most frequent neurologic symptom was headache (in $71 \%$ ), and the most common sign was facial palsy (14\%). Sleep disturbance was reported in $7 \%$, papilledema was present in $6 \%$, diplopia in $2 \%$, and 1 had a Guillain-Barrelike syndrome with a CSF protein of $231 \mathrm{mg} / \mathrm{dL}$. Elevations in CSF protein (32$58 \mathrm{mg} / \mathrm{dL}$ ) were found in 25 of 53 patients examined, and a mild lymphocytic pleocytosis in 15. Neurologic syndromes included encephalopathy, lymphocytic meningitis, cranial neuropathy, and pseudotumor cerebri. (Belman AL et al. Neurologic manifestations in children with North American Lyme disease. Neurology Dec 1993; $\underline{43}$ :2609-2614). (Reprints: Dr AL Belman, Department of Neurology, HSC T-12-020, SUNY at Stony Brook, Stony Brook, NY 11794).

COMMENT. The clinical course of Lyme disease in most children in this study was milder and shorter than that reported for adults, and meningoradiculitis (Bannwarth's syndrome) and peripheral neuropathy syndromes were rare. A pseudotumor cerebri syndrome appears to be unique to childhood Lyme disease. A first report of stroke caused by Lyme disease in North America involved a woman of 56 years

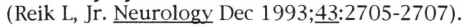

